\title{
Simulating fiber motion in the airflow in a rotor spinning unit
}

\author{
Huiting Lin ${ }^{1}$, Nicholus Tayari Akankwasa ${ }^{1}$ and Jun Wang ${ }^{1,2, a}$ \\ ${ }^{1}$ College of Textiles, Donghua University, Shanghai 201620, China \\ ${ }^{2}$ Key Laboratory of Textile Science \& Technology, Ministry of Education, Donghua University, Shanghai 201620, \\ China
}

\begin{abstract}
In this study, a numerical model for simulation of fiber motion in the transport channel of a rotor spinning unit has been developed. A chain represents the fiber consisting of a series of cylindrical rods connected with beads. The fiber model is applied on the turbulent airflow field of the transport channel to present fiber configuration at different times. Results show that the airflow field in the transport channel has a great impact on fiber configuration. The vortex generated in the channel inlet tends to cause a hook on the fiber leading end. As the leading end escapes from the vortex and moves downwards the transport channel, the accelerating air will facilitate fiber straightening.
\end{abstract}

\section{Introduction}

Air technology is widely used in the manufacture of textile. Especially in the rotor spinning process, it is used to transport and orientate fibers, which is a complex two-phase flow problem. In the study of two-phase (fluid/solid) problem, two basic approaches are used. One is the Eulerian approach, in which the solid phase is regarded as a continuous phase as the fluid phase. In this case, the fibers need to be treated as particles. However, as the fiber is elastic and flexible with a large aspect ratio, it cannot be considered as particles. Another is the Lagrangian approach, which treats the solid phase as discrete phase and the fluid phase as a continuous phase. The latter is usually used to study fluid/fiber interaction.

The key to study air/fiber interaction is to construct fiber mechanical model. Smith and Roberts [1] assumed the fiber as a chain composed of both spherical and cylindrical elements. The fiber model considering fiber stiffness was then applied on a laminar airflow. Reported by Bangert and Sagdeo [2], the fiber was represented by a rigid cylinder. However, their model cannot present fiber flexibility. Yamamoto and Matsuoka [3] modelled the motion of rigid and flexible fibers in a simple shear flow, and the fiber was composed of a series of contiguous spheres. The fiber could stretch, bend and twist by changing the bond distance, bond angle and torsion angle respectively between the neighbour spheres. However, it was computationally expensive when modelling a long fiber. Kong and Platfoot $[4,5]$ proposed a model represented by discrete nodes held together by weightless chains. Nevertheless, this model would not give a clear picture of elastic deformation of the fiber. Zeng et. al [6] established a bead-elastic rod model for simulating the fiber motion in high-speed air flow.

In rotor spun yarn spinning process, the air phase flow characteristics inside the rotor spinning unit was affected by the variations of structure and spinning parameters [7-9]. The airflow has a critical

\footnotetext{
${ }^{a}$ Corresponding author: junwang@dhu.edu.cn
} 
impact on yarn properties through influencing the configuration of fibers in yarns. Kong and Platfoot [8] simulated the two-dimensional airflow field in the transport channel. Affected by the channel geometry, a vortex was generated at the channel inlet. The vortex may cause fiber bending and hence deteriorate yarn properties. However, there is still a lack of studies on the relationship between the airflow and fiber configuration in rotor spinning. Therefore, the present paper will focus on studying the air/fiber interaction in the rotor spinning unit. The aim is to provide a theoretical guidance for rotor spun yarn production.

\section{Theoretical model}

The fiber/air interaction is assumed to be a one-way coupling model in which the presence of the fiber is assumed to have a negligible effect on the airflow field. Fiber trajectories can be obtained by applying the fiber model on the airflow field. Therefore, the airflow and fiber models will be constructed separately.

\subsection{Construction of the airflow model}

In rotor spinning, fiber slivers fed by the feeding roller are combed by the rotating opening roller into individual fibers. As the air inside the rotor is sucked by a pump, the pressure differential between the rotor and the trash box and the yarn guiding mouth forces the ambient air flowing into the rotor through the transport channel. Therefore, the individual fibers are transported into the rotor groove through the transport channel by the airflow, which will greatly influence fiber straightness. The fibers are then collected in the rotor groove and finally twisted into yarn by the rotating rotor.

Figure 1 presents the rotor spinning unit model and its main dimensions, including the rotor, the yarn guiding mouth, the transport channel, the opening roller and the trash box. As the fiber feeding channel is always blocked with fibers so that the air could hardly enter, this channel is not considered.

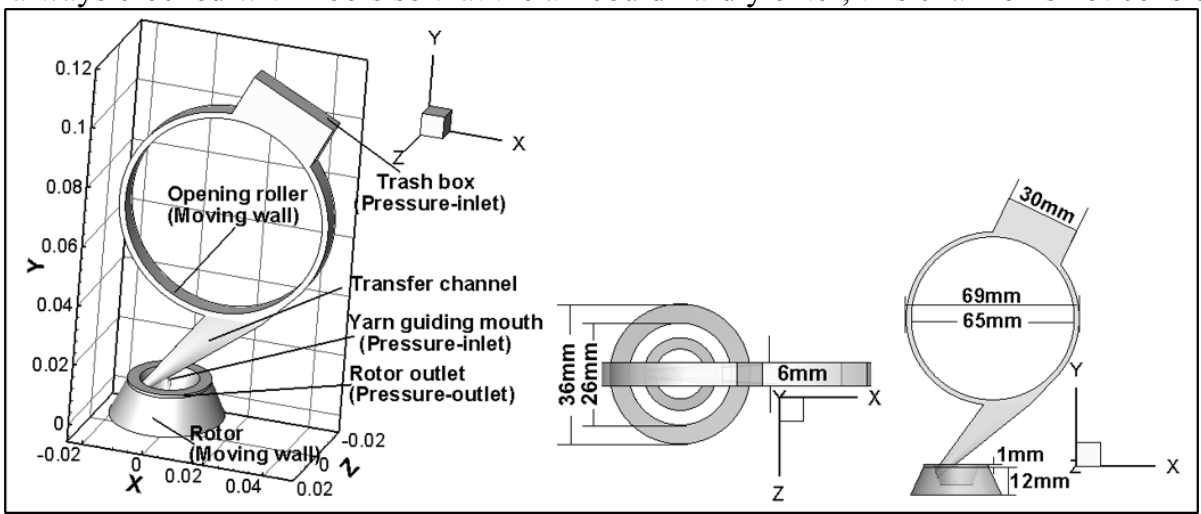

Figure 1. Geometry and dimensions of the rotor spinning unit.

Since the Reynolds number in the rotor spinning unit is high (up to 6000) [5], the airflow needs to be assumed as turbulent. As a complete spinning process is finished in a short time, a threedimensional, steady, viscous turbulence flow is considered. When considering $k-\varepsilon$ turbulence model, the general governing equation is:

$$
\frac{\partial(\rho \varphi)}{\partial t}+\operatorname{div}(\rho \mathbf{u} \varphi)=\operatorname{div}(\Gamma \operatorname{grad} \varphi)+S
$$

where $\rho$ is the fluid density, and $\mathbf{u}$ is a velocity vector. $\varphi$ represents a generic variable. $\Gamma$ is the diffusion coefficient and $S$ is the general source term. The different terms of the governing equations are illustrated in Table 1. $p$ is pressure. $u, v$ and $w$ are the velocity components in $x, y$ and $z$-direction, respectively. $\mu_{\text {eff }}$ is the effective viscosity, which is the sum of the fluid viscosity $\mu$ and the Reynolds apparent viscosity $\mu_{t}$. The turbulent kinetic energy $k$ and dissipation rate $\varepsilon$ are related to $\mu_{t}$ by the 
expression $\mu_{t}=\rho C_{\mu} k^{2} / \varepsilon . G_{k}$ is the generation of turbulent kinetic energy $k$ due to the mean velocity gradients. $\sigma_{k}$ and $\sigma_{\varepsilon}$ represent the turbulent Prandtl numbers corresponding to $k$ and $\varepsilon . C_{\mu}, C_{1 \varepsilon}$ and $C_{2 \varepsilon}$ are empirical constants.

A mix of structured hexahedral and unstructured tetrahedral grids are generated in the whole computational domain. The simulation is conducted using a commercial CFD package FLUENT. The finite volume discretization method is used and the second-order-upwind scheme is adopted due to its high accuracy to solve the governing equations.

Table 1. Terms of the governing equations considering $k-\varepsilon$ model.

\begin{tabular}{|c|c|c|c|}
\hline Equation & $\psi$ & Diffusivity $\Gamma$ & Source term $S$ \\
\hline Continuity & 1 & 0 & 0 \\
\hline$x$-momentum & $u$ & $\mu_{e f f}=\mu+\mu_{t}$ & 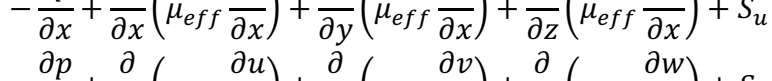 \\
\hline$y$-momentum & $v$ & $\mu_{\text {eff }}=\mu+\mu_{t}$ & $\begin{array}{c}-\frac{\overline{\partial y}}{\partial y}\left(\overline{\partial x}\left(\mu_{\text {eff }} \overline{\partial y}\right)+\frac{\overline{\partial y}}{\mu_{\text {eff }} \overline{\partial y}}\right)+\overline{\partial z}\left(\mu_{\text {eff }} \overline{\partial y}\right)+\mathrm{S}_{v} \\
\partial p\end{array}$ \\
\hline$z$-momentum & $w$ & $\mu_{e f f}=\mu+\mu_{t}$ & $\overline{\partial z}+\overline{\partial x}\left(\mu_{\text {eff } \overline{\partial z}}\right)+\overline{\partial y}\left(\mu_{\text {eff } \overline{\partial z}}\right)+\overline{\partial z}\left(\mu_{\text {eff } \overline{\partial z}}\right)$ \\
\hline Kinetic energy & $\kappa$ & $\mu+\mu_{t} / \sigma_{k}$ & $G_{k}+\rho \varepsilon$ \\
\hline Dissipation rate & $\varepsilon$ & $\mu+\mu_{t} / \sigma_{\varepsilon}$ & $(\varepsilon / k)\left(C_{1 \varepsilon} G_{k}-C_{2 \varepsilon} \rho \varepsilon\right)$ \\
\hline
\end{tabular}

\subsection{Construction of a fiber model}

The fiber is assumed to move freely in the air without being stretched, compressed and twisted. The fiber is a chain composed of $n$ beads and $n-1$ massless cylindrical rods, see Figure 2. The cylindrical rods have the same diameter as the beads. Only the beads are affected by forces, while the rods maintain the fiber configuration. The spatial positions of the beads represent the trajectory of the fiber.

In the fiber model, bead $i$ only connected with bead $i-1$ and $i+1$. Therefore, bead 1 and bead $n$ become the first and last beads, respectively. The mass of bead $i m_{i}$ is described as:

$$
m_{i}=0.5 * \rho_{f}\left(l_{(i-1, i)}+l_{(i, i+1)}\right)(i=1,2,3 \ldots \ldots \mathrm{n})
$$

where $\rho_{f}$ is the fiber density. $l_{(i-1, i)}$ and $l_{(i, i+1)}$ are the length of fiber section $(i-1, i)$ and $(i, i+1)$, respectively. For beads 1 and $n+1$, the mirror reflection is used, therefore, $l_{(0,1)}=l_{(1,2)}$ and $l_{(n-1, n)}=$ $l_{(n, n+1)}$ are obtained.

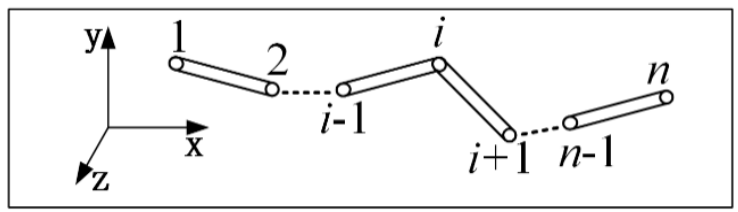

Figure 2. Diagram of the fiber model.

According to Newton's second law, the equation for the motion of the beads that constitute the fiber is:

$$
m_{i} \frac{\mathrm{d}^{2} \mathbf{r}_{i}}{\mathrm{~d} t}=\mathbf{F}_{b i}+\mathbf{F}_{d i}
$$

$\mathbf{r}_{i}$ represents the position of bead $i$ in the airflow field, which can be expressed as $\mathbf{r}_{i}=x_{i}(t) i+$ $y_{i}(t) j+z_{i}(t) k . \mathbf{F}_{b i}$ and $\mathbf{F}_{d i}$ are respectively the bending force and the drag force exerted on bead $i$.

The angle $\theta_{i}$ between section $(i-1, i)$ and $(i, i+1)$ represents that section $(i, i+1)$ is subjected to the bending force (see Figure 3). The bending torque $\mathbf{M}_{b i}$ acted on bead $i$ is:

$$
\mathbf{M}_{b i}=-k_{b} \theta_{i}
$$

where $k_{b}$ is the bending constant related to fiber elasticity modulus $E$ and inertia moment of the fiber cross section to the central axis $I_{b}$, which are:

$$
k_{b}=2 E I_{b} / l_{(i, i+1)} \quad \text { and } \quad I_{b}=\pi d_{f}^{4} / 64
$$


where $d_{f}$ is the fiber diameter. $\theta_{i}$ can be obtained by the following equation:

$$
\operatorname{Cos} \theta_{i}=\mathbf{e}_{i} \mathbf{e}_{i+1}
$$

$\mathbf{e}_{i}$ and $\mathbf{e}_{i+1}$ are the unit vectors of section $(i-1, i)$ and $(i, i+1)$.

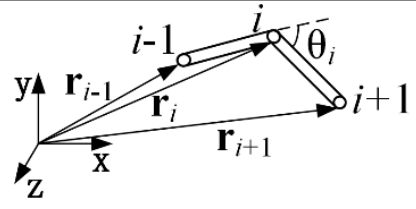

Figure 3. Sketch of fiber bending.

The bending force $\mathbf{F}_{b i}$ exerted on bead $i$ can be expressed as:

$$
\mathbf{F}_{b i}=\mathbf{M}_{b i} / l_{(i, i+1)}
$$

$\mathbf{F}_{b i}$ is perpendicular to $\mathbf{e}_{i+1}$ with its direction to the equilibrium position.

When immersed in the airflow, the fiber is subjected to the aerodynamic forces. Only the drag force is considered in this paper. The drag force is related to the air characteristics, fiber shape and fiber orientation. If considering section $(i, i+1)$, the drag force $\mathbf{F}_{d i}$ is:

$$
\mathbf{F}_{d i}=(\pi / 8) \rho_{a} d_{s p h i}^{2} C_{d}\left(\mathbf{V}_{a i}-\mathbf{V}_{f i}\right)\left|V_{a i}-V_{f i}\right|
$$

where $\rho_{a}$ is the air density and $d_{s p h i}$ is the equivalent spherical diameter of section $(i, i+1)$.

$$
d_{s p h i}=\sqrt[3]{1.5 d_{f}^{2} l_{(i, i+1)}}
$$

$\mathbf{V}_{f i}$ and $\mathbf{V}_{a i}$ are the velocities of bead $i$ and the air in the position of bead $i$. The direction of $\mathbf{F}_{d i}$ is the same as the relative velocity $\mathbf{V}_{a i}-\mathbf{V}_{f i}$. $C_{d}$ is the drag coefficient. The expression to determine $C_{d}$ proposed by Andrea and Martin [10] is adopted, which is described as:

$$
C_{d}=\frac{8}{R e} \frac{1}{\sqrt{\emptyset_{\perp}}}+\frac{16}{R e} \frac{1}{\sqrt{\emptyset}}+\frac{3}{R e} \frac{1}{\emptyset^{0.75}}+0.4210^{0.4(-\log \emptyset)^{0.2}} \frac{1}{\emptyset_{\perp}}
$$

where $\emptyset$ and $\emptyset_{\perp}$ are sphericity and crosswise sphericity. They are given as:

$$
\emptyset=s / s_{f i} \quad \text { and } \quad \emptyset_{\perp}=s_{m} / s_{\perp}
$$

$s$ is the surface of a sphere having the same volume as section $(i, i+1)$ and $s_{f i}$ is the surface of section $(i, i+1) . s_{m}$ is the maximum cross-sectional area of the volume equivalent sphere. $s_{\perp}$ is the projected cross-sectional area of section $(i, i+1)$ perpendicular to the airflow. They are expressed as:

$$
s=\pi d_{s p h i}^{2} \quad \text { and } \quad s_{f i}=\pi d_{f} l_{(i, i+1)} \quad \text { and } \quad s_{m}=0.25 \pi d_{s p h i}^{2}
$$

$s_{\perp}$ is related to the relative position between section $(i, i+1)$ and air velocity vector. Figure 4 illustrates the three possible cases of the relative position.

(a) Air velocity vector is perpendicular to the central axis of section $(i, i+1)$, then $s_{\perp}=d_{f} l_{(i, i+1)}$.

(b) Air velocity vector is parallel to the central axis of section $(i, i+1)$, then $s_{\perp}=0.25 \pi d_{f}^{2}$.

(c) The angle between air velocity vector and the central axis of section $(i, i+1)$ is $\alpha_{i}$, then $s_{\perp}=d_{f} l_{(i, i+1)} \sin \alpha_{i}$.

For section $(i, i+1)$, the Reynolds number $R e$ is:

where $\mu_{a}$ is the air dynamic viscosity.

$$
R e=\rho_{a}\left(V_{a i}-V_{f i}\right) d_{s p h i} / \mu_{a}
$$

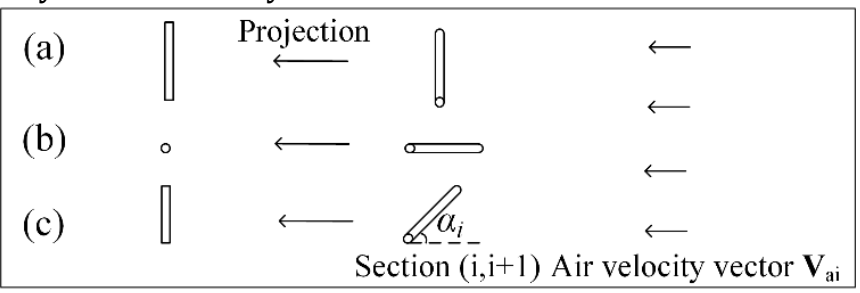

Figure 4. Relative position between section $(i, i+1)$ and the air velocity vector. 


\subsection{Simulation procedure}

The following constraint condition needs to be satisfied during calculation:

$$
\left|\mathbf{r}_{i+1}-\mathbf{r}_{i}\right|=l_{(i, i+1)}
$$

As equation (14) may be broken due to the numerical error, a procedure is conducted for adjustment at each time step. The position of bead $i$ is adjusted from $\mathbf{r}_{i}$ to $\mathbf{r}_{i}+\Delta \mathbf{r}_{i}(\mathrm{i}=1,2, \ldots, \mathrm{n})$. $\Delta \mathbf{r}_{i}$ can be obtained by solving the quantity as minimum: $\delta=\sum\left(\Delta \mathbf{r}_{i}\right)^{2}[11]$.

The procedure for the solution is, (a) Calculate the air flow field of the rotor spinning unit without the fiber by equation (1), then the air velocity can be obtained; (b) For a given fiber, calculate equations (4) - (7), $\mathbf{F}_{b i}$ is obtained; (c) Calculate equations (8) - (13), $\mathbf{F}_{d i}$ is obtained; (d) Solve equations (2) - (3), the position $\mathbf{r}_{i}^{\prime}$ and velocity $\mathbf{V}_{f i}^{\prime}$ of bead $i$ at $t^{\prime}=t+\Delta t$ are obtained; (f) If $\mathbf{r}_{i}^{\prime}$ does not satisfied the constraint condition (equation (14)), then the procedure is applied to adjust the position; (e) Repeat steps (a) - (f), the trajectory of a fiber as a function of time is obtained.

\section{Results and discussion}

The material parameters for the fiber used are diameter of $0.02 \mathrm{~mm}$, linear density of 1.8 dtex, elastic modulus of $8 \mathrm{GPa}$ and length of $12 \mathrm{~mm}$. Initially, the fiber is assumed to be straight, and the fiber is parallel to the transport channel inlet cross section, see Figure 5 (b). The initial fiber velocity is assumed to be $5 \mathrm{~m} / \mathrm{s}$. The calculation ends once the fiber collides with the walls or the leading end of the fiber enters the rotor.

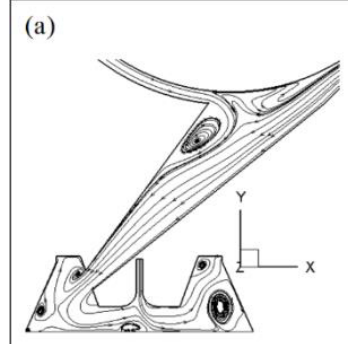

(d)

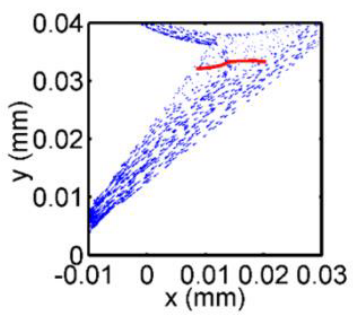

(g)

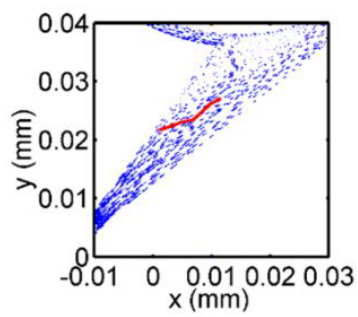

(b)

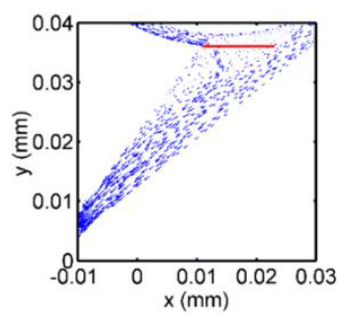

(e)

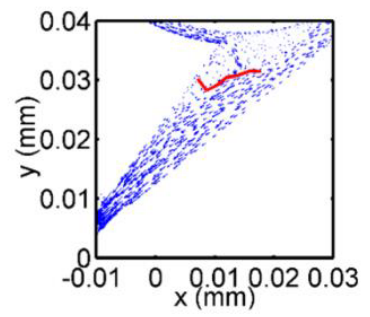

(h)

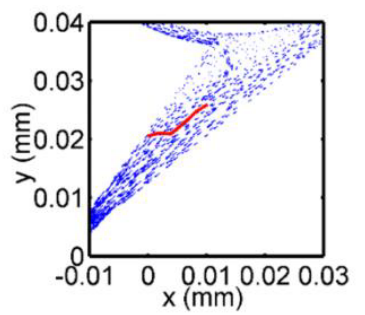

(c)

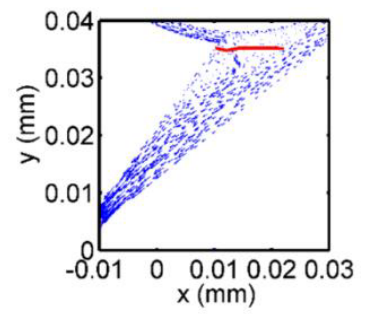

(f)

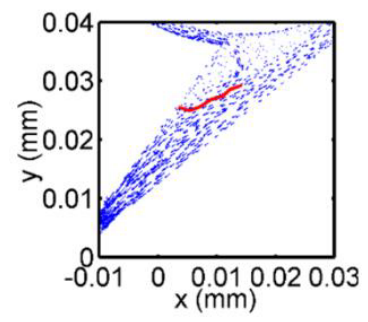

(i)

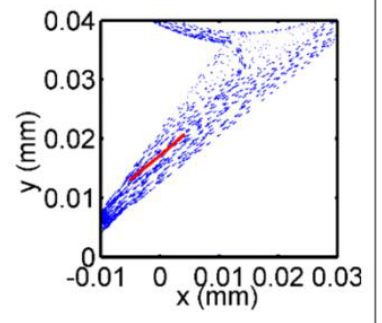

Figure 5. (a) Velocity streamline and (b) - (i) velocity vector and fiber configuration in the transport channel at plane $\mathrm{z}=0 \mathrm{~mm}$. The time step for (b) - (i) is $4 \times 10^{-4} \mathrm{sec}$; figure (b) shows the initial position of the fiber $(t=0$ $\mathrm{sec})$. 
Figure 5(a) shows the velocity streamline in the transport channel and rotor. The air from the opening roller chamber enters the rotor through the transport channel, at which a vortex is generated. Figures 5(b) - (i) illustrate the fiber trajectories along the transport channel at different times. The variations of fiber configuration indicate that the airflow inside the rotor spinning unit is critical to the fiber straightness. The fiber moves downwards with its leading end entering the vortex (Figure 5(c)). The fiber leading end is gradually bent due to the vortex. However, the intensity of the vortex is not strong enough to drag the whole fiber into the vortex. The leading end of the fiber moves faster than its trailing end, therefore, as soon as its leading end escapes from the vortex, the fiber moves along the transport channel longitudinal axis. Figure 5(g) - (i) shows that the fiber undergoes straightening gradually as it is moving down, indicating that the converging transport channel along with the inside accelerating air is helpful in straightening the hooked fibers.

\section{Conclusion}

In this paper, the simulation of fiber motion in the turbulent airflow field was carried out. The fiber was modelled as a bead-cylindrical rod chain and was applied in the transport channel airflow field in the rotor spinning machine. The airflow field has a critical influence on fiber configuration. The fiber tends to bend when it runs into a vortex. However, it will escape from the vortex as long as the vortex is not strong enough to trap it. As the fiber moves downwards the transport channel, the accelerating air facilitates fiber-straightening, eliminating the temporary bending caused by the vortex.

\section{Acknowledgement}

This work was supported by the Key Grant Project of Chinese Ministry of Education (Grant 113027A).

\section{References}

1. A.C. Smith, W.W. Roberts, Text. Res. J. 64, 335 (1994).

2. L.H. Bangert, P.M. Sagdeo, Text. Res. J. 47, 773 (1977).

3. S. Yamamoto, T. Matsuoka, J. Chem. Phys. 98, 644 (1993).

4. L.X. Kong, R.A. Platfoot, Text. Res. J. 67, 269 (1997).

5. L.X. Kong, R.A. Platfoot, Comput. Struct. 78, 237 (2000).

6. Y.C. Zeng, J.P. Yang, C.W. Yu, Appl. Math. Model. 29, 253 (2005).

7. H.T. Lin, Y.C. Zeng, J. Wang, Text. Res. J. 86, 115(2015).

8. L.X. Kong, R.A. Platfoot, Text. Res. J. 66, 641 (1996).

9. L.H. Zhang, B.X. Zhang, J. China Text. Univ. 17, 16 (1991).

10. A. Hölzer, M. Sommerfeld, Powder Technol. 184, 361 (2008).

11. M. Doi, D. Chen, J. Chem. Phys. 90, 5271 (1989). 